\title{
Open versus endovascular repair for patients with acute traumatic rupture of the thoracic aorta
}

\author{
Ludovic Canaud, MD, Pierre Alric, MD, PhD, Pascal Branchereau, MD, Frédéric Joyeux, MD, \\ Kheira Hireche, MD, Jean-Philippe Berthet, MD, and Charles Marty-Ané, MD, PhD
}

\begin{abstract}
Objective: The study objective was to compare the outcome between open and endovascular repair of acute traumatic rupture of the thoracic aorta.

Methods: Seventy-five patients (mean age $38.6 \pm 10.7$ years) with an acute traumatic aortic rupture were referred to the Arnaud de Villeneuve Hospital between January 1990 and January 2010. Between January 1990 and December 2000, 35 patients (33 men, mean age $35.8 \pm 11.3$ years) underwent surgical repair using cardiopulmonary bypass. From January 2001, an endovascular approach was deliberately chosen; 40 patients (30 male, mean age $41 \pm 10.1$ years) underwent endovascular repair. The 2 groups were statistically comparable.
\end{abstract}

Results: The overall mortality rates for the surgical and endovascular groups were $11.4 \%$ (intraoperative mortality: $8.5 \%$ ) and $2.5 \%$ (intraoperative mortality: $0 \%$ ), respectively. The mortality rates related to aortic repair for the surgical and endovascular groups were $11.4 \%$ and $0 \%$, respectively. In the surgical group, the morbidity rate was $14.2 \%$ : 4 cases of recurrent nerve palsy and 1 case of false anastomotic aneurysm were diagnosed at 52 months. In the endovascular group, the morbidity rate was $20 \%: 3$ cases of intraoperative inadvertent coverage of supra-aortic trunks (requiring a secondary procedure in 2 cases after 1 and 2 years to revascularize the supra-aortic trunks), 1 proximal type I endoleak (requiring deployment of a second stent-graft at day 2), 2 stentgraft collapses in the first postoperative month (treated by open repair and explantation in 1 case and by the deployment of a second stent-graft in 1 case), 1 vertebrobasilar insufficiency after left subclavian artery coverage, and 1 intraoperative iliac rupture (surgically repaired). No cases of paraplegia or stroke were observed. The median follow-up was 7.7 (range, 0.4-15) years.

Conclusions: Compared with open repair, endovascular repair of traumatic thoracic aortic rupture is associated with a lower death rate but failed to reach statistical significance, most likely because of underpowering. These results prompt us to consider endovascular repair as the first-line therapy for acute traumatic rupture of the thoracic aorta, except in some rare but challenging anatomic situations. New stent-graft designs, sizes, and deployment systems could improve the results of endovascular repair in these indications. (J Thorac Cardiovasc Surg 2011;142:1032-7)

Open operative repair of a traumatic thoracic aortic rupture in the presence of other associated injuries correlates with significant mortality approaching $8 \%$ to $15 \%$, despite significant improvements in intensive care. ${ }^{1}$ Paraplegia rates of $2.3 \%$ to $14 \%$ remain high as the result of aortic thoracic crossclamping and prolonged distal hypoperfusion in polytraumatized patients, despite the use of circulatory assistance. $^{2}$ Moreover, these patients typically have other severe injuries, and the use of extracorporeal circulation, particularly the use of systemic heparinization, complicates the management of those associated injuries.

From the Department of Vascular and Thoracic Surgery, Arnaud de Villeneuve Hospital, Montpellier, France.

Disclosures: Authors have nothing to disclose with regard to commercial support. Received for publication Sept 20, 2010; revisions received Nov 4, 2010; accepted for publication Nov 19, 2010; available ahead of print March 14, 2011.

Address for reprints: Ludovic Canaud, MD, Service de Chirurgie Vasculaire et Thoracique, Hôpital A de, Villeneuve, 191 av Doyen Gaston Giraud, 34090 Montpellier,

France (E-mail: ludoviccanaud@hotmail.com).

$0022-5223 / \$ 36.00$

Copyright $(c) 2011$ by The American Association for Thoracic Surgery doi:10.1016/j.jtcvs.2010.11.051
In 1994, Dake and colleagues ${ }^{3}$ reported the first successful endovascular repair of thoracic aorta disease. Currently, stent-graft placement represents a valid option for thoracic aortic aneurysms and complicated type B dissections, especially for patients at high surgical risk.

Despite lack of level I evidence, endovascular treatment of traumatic thoracic aortic rupture is increasingly being used as the primary modality of therapy. The purpose of this retrospective and monocentric study was to evaluate outcomes of open and endovascular repair of traumatic thoracic aortic rupture.

\section{MATERIALS AND METHOD}

Institutional review board approval was obtained for the review of all patient records relevant to this study.

\section{Patients}

Seventy-five patients (mean age, $38.6 \pm 10.7$ years; range, $15-78$ years) with an acute traumatic rupture of the thoracic aorta were referred to the Arnaud de Villeneuve Hospital between January 1990 and January 2010, 63 of whom were male $(84 \%)$. 


\section{Abbreviations and Acronyms \\ AAST $=$ American Association for the Surgery of Trauma \\ $\mathrm{BT}=$ brachiocephalic trunk \\ $\mathrm{CT}=$ computed tomography \\ LCCA $=$ left common carotid artery \\ LSA = left subclavian artery}

All patients admitted for blunt traumatic injury during that time were included. Diagnosis of aortic disruption was achieved by a preprocedural contrast-enhanced computed tomography (CT) scan of all patients. Subcategories of aortic lesions in the endovascular group and the open repair group were pseudoaneurysm (17 and 18, respectively), intramural hematoma (18 and 13 , respectively), and free rupture (5 and 4 , respectively).

To define the severity of trauma in the conventional surgical group and endovascular stent group, an anatomic scoring system, the injury severity score from 0 to 75 , was used. ${ }^{4}$

\section{Therapeutic Strategy}

Between January 1990 and December 2000, all patients underwent surgical repair using cardiopulmonary bypass. From January 2001, an endovascular approach was deliberately chosen for all patients.

We aimed to perform surgical or endovascular repair soon after admission, unless severe associated injuries or complications restricted this policy. In 9 patients (endovascular in 6, surgical repair in 3), treatment was delayed for more than 5 days after trauma caused by septic state, major cerebral lesions, or severe pulmonary contusion (Table 1).

\section{Surgical Approach}

In all patients, surgical repair was performed using a standard left heart bypass driven with a Bio-Medicus pump (Bio-Medicus Inc, Eden Prairie, Minn). This circulatory support was inserted between the left atrium and the left common femoral artery. In the absence of associated bleeding injuries, a bolus of $0.5 \mathrm{mg} / \mathrm{kg}$ of heparin was given. The thoracic aorta was approached through a left posterolateral thoracotomy. The aorta was repaired with direct suturing or prosthetic graft interposition.

\section{Endovascular Repair}

Suitable morphology for stent-graft placement requires a proximal aortic neck length of at least $15 \mathrm{~mm}$. Measurements from preprocedural imaging data were used to select the appropriate diameter and length of the stent-graft. Devices were oversized by $10 \%$ to $20 \%$ greater than the minor axis of the aortic neck to provide sufficient radial force for adequate fixation.

TABLE 1. Patient population and associated injuries

\begin{tabular}{lccc}
\hline & $\begin{array}{c}\text { Surgical } \\
\text { group }\end{array}$ & $\begin{array}{c}\text { Endovascular } \\
\text { group }\end{array}$ & $\boldsymbol{P}$ \\
\hline Gender (m/f) & $33 / 2$ & $30 / 10$ & .3 \\
Age (y) & 35.8 & 41 & .4 \\
Delay between trauma & $3.4(0-28)$ & $7.3(0-30)$ & .08 \\
$\quad$ and treatment (d/median) & & & \\
ISS (median) & $37 \pm 5$ & $34 \pm 7$ & .6 \\
Associated injuries & & & \\
$\quad$ Thoracic injuries (\%) & 94.2 & 92.5 & \\
$\quad$ Cranial and spinal injuries (\%) & 42.5 & 37.1 & \\
$\quad$ Abdominal injuries (\%) & 45.7 & 55 & \\
\hline
\end{tabular}

ISS, Injury severity score.
All procedures were performed in the operating room under general anesthesia. All angiograms were performed through a $5 \mathrm{~F}$ calibrated pigtail catheter (Cook Australia Pty Ltd, Queensland, Brisbane, Australia) placed percutaneously into the aortic arch via a brachial artery. A $260-\mathrm{cm}$, 0.035-inch Terumo guidewire (Terumo Medical Corporation, Tokyo, Japan) was placed under fluoroscopic control into the ascending aorta through a sheath in the common femoral artery; a $5 \mathrm{~F}$ measuring pigtail catheter was advanced into the ascending aorta over the Terumo guide. This pigtail catheter was used to exchange the Terumo guide wire for a 0.035-inch-diameter Lunderquist (Cook Inc, Bloomington, Ind) to guide passage of the $22 \mathrm{~F}$ to $24 \mathrm{~F}$ sheath. In absence of associated bleeding injuries, a bolus of $0.5 \mathrm{mg} / \mathrm{kg}$ of heparin was given. Angiography was performed before stent-graft deployment. Stent-graft deployment was performed under fluoroscopic control. A control angiography was performed to confirm appropriate position of the device and exclusion of the aortic disruption. In case of endoleak after stent-graft deployment, the stent-graft was further expanded with a low-pressure balloon.

Follow-up surveillance was performed with serial CT scans at 1 week; at 3,6 , and 12 months; and annually thereafter. Outcomes were analyzed using Kaplan-Meier life-table analysis.

\section{Statistical Analysis}

Categoric data are presented as frequency (percentage), and continuous data are presented as mean + standard deviation or median with interquartile ranges. Comparison of continuous data was by the Student $t$ test for normally distributed data and Mann-Whitney test for non-normally distributed data. Comparison of categoric data was by chi-square test for normally distributed data and Fisher exact test for non-normally distributed data.

\section{RESULTS \\ Follow-up}

No patients have been lost to follow-up, and all have completed each of their scheduled follow-up evaluations and CT scans.

\section{Surgical Group}

In our series, 35 patients ( 33 male; mean age, $35.8 \pm 11.3$ years) underwent surgical repair of a traumatic rupture of the thoracic aorta. The aorta was repaired with direct suturing in 13 patients $(37 \%)$ and with a prosthetic graft interposition in 22 patients $(63 \%)$. The intraoperative mortality was $8.5 \%(n=3)$ : these 3 patients had a free rupture of the aorta. The cause of the intraoperative mortality was irreversible cardiac arrest in all 3 patients. The overall mortality was $11.4 \%(n=4)$. One patient died of acute respiratory distress syndrome on the sixth postoperative day. No patients experienced stroke or paraplegia.

The morbidity rate was $14.2 \%(n=5): 4$ cases of recurrent nerve palsy and 1 false anastomotic aneurysm diagnosed at 52 months and treated conservatively.

The median follow-up for survivors was 13 years (range, 9-20 years). During follow-up, no patient required a redo operation.

\section{Endovascular Group}

In our series, 40 patients (30 male; mean age, $41 \pm 10.1$ years) underwent endovascular repair for a traumatic rupture of the thoracic aorta. Technical success was obtained 
in all the patients. The TAG stent-graft (WL Gore \& Associates, Flagstaff, Ariz) was used in 18 cases, the Talent stent-graft (Medtronic Vascular, Santa Rosa, Calif) was used in 9 cases, the Valiant stent-graft (Medtronic Vascular) was used in 11 cases, and a Zenith distal extension cuff (Cook Inc) was used in 2 cases. The mean diameter of the implanted stent-graft was $27 \pm 4 \mathrm{~mm}$ (range, 18-40 mm), the mean distal aortic diameter was $19.8 \pm 4.5 \mathrm{~mm}$, and the mean stent-graft length was $106 \pm 22 \mathrm{~mm}$ (range, 100-200 mm).

A retroperitoneal iliac approach was necessary in 4 cases where progression of the sheath by femoral access was impossible. Technical success was achieved in all cases. In 8 patients, for whom proximal aortic neck length was insufficient, the ostium of the left subclavian artery (LSA) was totally covered deliberately. For 1 patient in whom the hemodynamic status was unstable at the time of aortic disruption, an LSA to the left common carotid artery (LCCA) transposition was also performed to treat vertebrobasilar insufficiency (vertigo and drop attacks). For 2 patients whose hemodynamic status was stable at the time of diagnosis, the landing zone was extended by prophylactic LSA to LCCA transposition before stent-graft repair. For 1 patient whose hemodynamic status was stable at the time of diagnosis, the landing zone was extended by prophylactic LCCA to brachiocephalic trunk (BT) transposition and LSA to LCCA transposition before stent-graft repair (Figure 1).

The intraoperative mortality was nil. The mortality rate related to aortic disruption was $0 \%$. The overall mortality

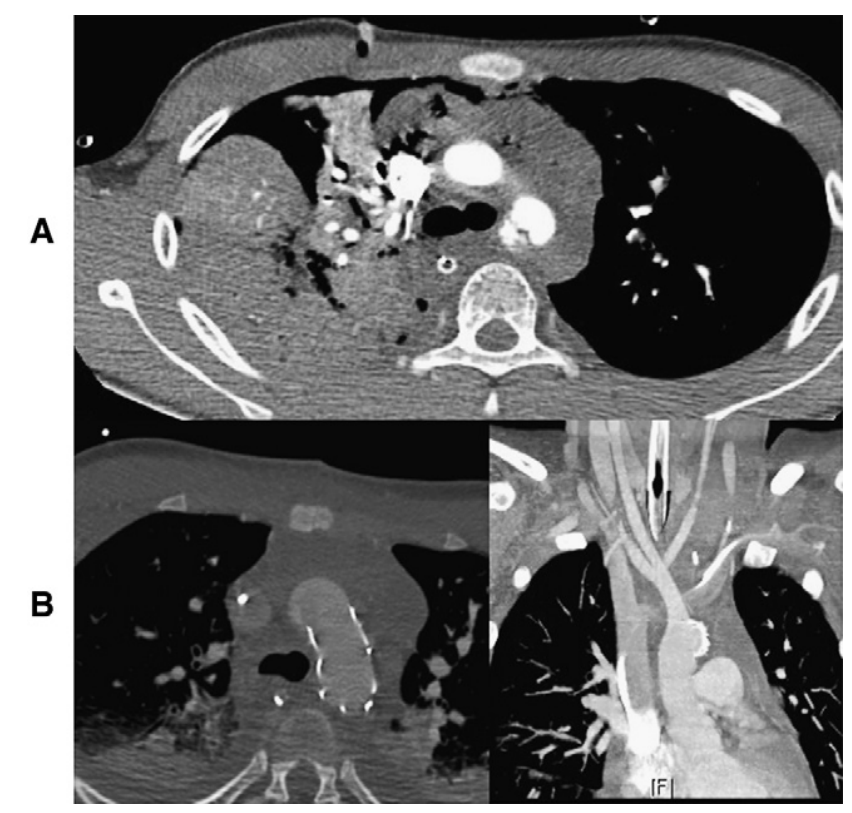

FIGURE 1. A, Acute rupture of the aortic arch between the LSA and the LCCA. B, Extension of the proximal landing zone by prophylactic LCCA to BT transposition and LSA to LCCA transposition before stent-graft repair. for the endovascular group was $2.5 \%(n=1)$. One patient died of a traumatic intracranial hematoma on the third postoperative day. The morbidity rate was $20 \%$ (Table 2 ). Three cases of inadvertent coverage of supra-aortic trunks occurred. In the first case, a proximal stent-graft migration totally covered the LCCA ostium. The stent-graft was pulled distally by traction from a low-pressure inflated balloon and reestablished flow to the LCCA, but an excessive distal migration led to a proximal type I endoleak detected on the postoperative CT scan. This endoleak was successfully treated by a proximal implantation of a second stent-graft on the second postoperative day. In the second case, a proximal stent-graft migration occurred, totally covering the LCCA and the BT. The flow was reestablished into the LCCA and the BT by transcarotid insertion of a selfexpanding stent with a high radial force alongside the thoracic stent-graft. After 1 year of follow-up, the stent placed in the LCCA completely collapsed, leading to a 70\% stenosis of the LCCA and BT (Figure 2). Therefore, an ascending aorta to BT and LCCA bypass was performed through a median sternotomy. In the third case, a partial coverage of the LCCA occurred, related to apposition of a scalloped flare of a TAG device in front of the ossium of the LCCA. Two years later, a duplex follow-up disclosed a significant stenosis of the LCCA. The flow was reestablished into the LCCA by transcarotid insertion of a balloon-expandable stent. One intraoperative iliac rupture occurred and was surgically repaired. One patient presented a vertebrobasilar insufficiency after deliberated coverage of the LSA and required an LSA transposition.

Two patients experienced a stent-graft collapse. In the first case, the collapse of the stent-graft was revealed at the 30th day after implantation by a pseudocoarctation syndrome leading to functional renal failure. Open explant and repair by a left thoracotomy were successfully performed. In the second case, the stent-graft collapse occurred 20 days after exclusion of traumatic rupture and was successfully reexpanded by deployment of a second stentgraft (Valiant stent-graft). No patients experienced stroke or paraplegia. The median follow-up for survivors was 3.1 years (range, $0.2-9$ years).

\section{Statistical Analysis}

There was no difference between the endovascular and open repair groups with respect to age $(32 \pm 12$ years vs $41 \pm 8$ years; $P=.4$ ) or the mean injury severity score ( $37 \pm 5$ vs $34 \pm 7 ; P=.6$ ), respectively (Table 1$)$. There was no difference in mortality between the endovascular and open repair groups $(P=.08)$ (Figure 3$)$.

\section{DISCUSSION}

For decades, the treatment of choice has been immediate repair by a left thoracotomy, aortic crossclamping and direct suturing, or prosthetic graft interposition. The outcome 
TABLE 2. Complications of endovascular repair

\begin{tabular}{|c|c|c|c|c|}
\hline & Type of graft & Complication & Delay (day) & Intervention \\
\hline $1-2$ & $\begin{array}{l}\text { TAG } 2 * 31 / 100 \mathrm{~mm} \\
\text { (WL Gore \& Associates, } \\
\text { Flagstaff, Ariz) }\end{array}$ & $\begin{array}{l}\text { Proximal endograft migration } \\
\text { totally covering the } \\
\text { LCCA ostium }\end{array}$ & Intraoperative & $\begin{array}{l}\text { Endograft pulled distally because of an } \\
\text { inflated low-pressure balloon; excessive } \\
\text { distal migration led to a proximal type I } \\
\text { endoleak successfully treated by a proximal } \\
\text { implantation of a second endograft }\end{array}$ \\
\hline 3 & TAG $31 / 100$ & $\begin{array}{l}\text { Complete coverage of the } \\
\text { LCCA and BT }\end{array}$ & Intraoperative & $\begin{array}{l}\text { Stenting of the LCCA across the ostium } \\
\text { with a nitinol stent Luminex } 8 / 80 \mathrm{~mm} \\
\text { Ascending aorta to BT and LCCA bypass }\end{array}$ \\
\hline 4 & TAG $26 / 100 \mathrm{~mm}$ & $\begin{array}{l}\text { Partial coverage of the } \\
\text { LCCA occurred }\end{array}$ & Intraoperative & $\begin{array}{l}\text { Transcarotid insertion of a } \\
\text { balloon-expandable stent }\end{array}$ \\
\hline 5 & $\begin{array}{l}\text { Valiant 34/100 (Medtronic } \\
\text { Vascular, Santa Rosa, Calif) }\end{array}$ & Iliac rupture & Intraoperative & Surgically repaired \\
\hline 6 & TAG $26 / 100 \mathrm{~mm}$ & Collapse of an endograft & 20 & Deployment of a second stent-graft \\
\hline 7 & TAG $26 / 100 \mathrm{~mm}$ & Collapse of an endograft & 30 & Open explant and repair by a left thoracotomy \\
\hline 8 & TAG $26 / 100 \mathrm{~mm}$ & $\begin{array}{l}\text { Vertebrobasilar insufficiency } \\
\text { after LSA coverage }\end{array}$ & 1820 & LSA transposition \\
\hline
\end{tabular}

$L C C A$, Left common carotid artery; $B T$, brachiocephalic trunk; $L S A$, left subclavian artery.

has been constantly improved, and in particular, the risk of paraplegia resulting from open surgery has been decreased to $2 \%$ by refinements in medical and surgical management of these patients by using surgical adjuncts (distal bypass), minimizing heparin, or delaying surgery with aggressive blood pressure control to allow stabilization of the patient. However, mortality remains high, with $12 \%$ to $26 \%$ due to associated injuries. ${ }^{5,6}$

There have been 2 large, prospective observational studies evaluating the management and outcomes of blunt thoracic artery injuries (American Association for the Surgery of Trauma $[\mathrm{AAST}] 1^{7}$ and AAST2 ${ }^{8}$ ). The most significant finding of this comparison is the marked change in operative management from exclusive open repairs $(100 \%)$ in the

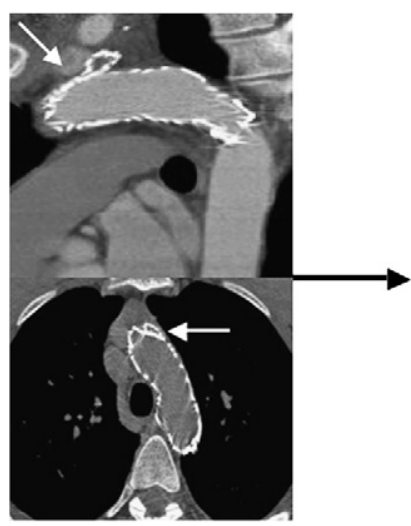

A

FIGURE 2. A, Complete collapse of a bare stent inserted alongside the thoracic stent-graft to reestablish blood flow into the supra-aortic trunks after inadvertent coverage of the supra-aortic trunks. B, An ascending aorta to BT and LCCA bypass was performed via a median sternotomy. early period to a majority of endovascular repairs $(65 \%)$ in the more recent series. The AAST2 study was a multicentered prospective study of 193 patients from 18 trauma centers. A total of 125 patients were treated with endovascular repair, and 68 patients were treated with open repair. This study showed a significant difference in mortality in favor of endovascular repair $(7.2 \%$ for endovascular repair vs $23.5 \%$ for open repair). Furthermore, a recent metaanalysis showed a significant reduction in mortality rates with $7.6 \%$ for endovascular repair versus $15 \%$ for open repair. Endovascular repair had improved paralysis rates at $0 \%$ compared with $18 \%$ for open repair. ${ }^{9}$ Our study showed that endovascular repair was associated with a strong trend

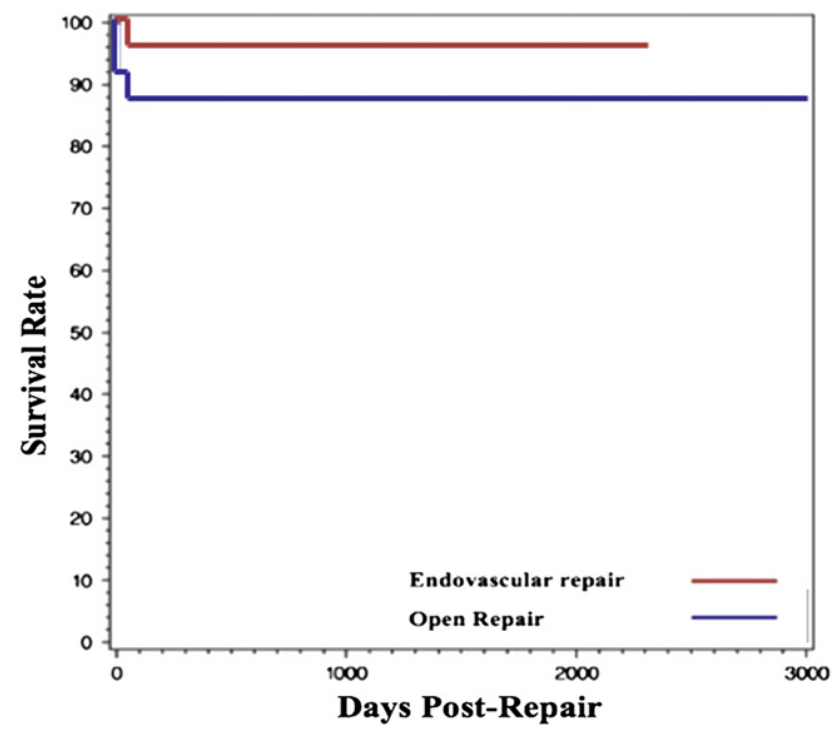

FIGURE 3. Actuarial survivals of patients. 
for lower mortality than open repair but failed to reach statistical significance $(P=.08)$, most likely because of underpowering.

Endovascular repair requires a proximal landing zone of at least $15 \mathrm{~mm}$ in length. Because of the proximity of these injuries to the LSA, the LSA has to be overstented. In hemodynamically unstable patients, the coverage of the LSA without prior revascularization is required to allow a swift procedure. LSA revascularization can be proposed in a second operative time in case of left arm ischemia or vertebrobasilar insufficiency. For hemodynamically stable patients, an LSA revascularization can be proposed before stent-graft repair; intraoperative angiography can be achieved by the left cervical approach. A meta-analysis of Peterson and coworkers ${ }^{10}$ showed that morbidity relative to LSA revascularization before stent-graft repair was lower than morbidity in cases of LSA coverage without revascularization ( $3 \%$ vs $23 \%$ ). Furthermore, Buth and associates ${ }^{11}$ assessed the significance of clinical factors that potentially influenced the occurrence of neurologic sequelae during endovascular repair of the thoracic aorta: Perioperative paraplegia or paraparesis was significantly associated with blockage of the LSA without revascularization.

Postponement of aortic repair have been advocated ${ }^{12}$ when severe concomitant lesions preclude safe immediate repair. Delayed treatment increases the risks of 3 major complications: an unforeseeable risk of delayed free rupture of an initially stable aortic tear; a progressive dilation of the involved aortic segment exerting compression on the trachea and the left main bronchus; and the creation of a fibrous and calcified connection within the aortic wall itself, potentially modifying aortic compliance and compromising the success of endovascular treatment. A controlled hypotension may cause cerebral hypoperfusion among patients who have often had a cerebral trauma. Furthermore, since the introduction of endovascular repair with stent-graft available on the shelf, there are not many indications to defer this low invasive approach. Endovascular procedure allows prompt exclusion of the aortic injury without thoracotomy and aortic clamping, both undesirable in patients with severe injuries. In our institution, we believe that early endovascular management offers important advantages over a deliberate wait.

In regard to the morbidity of our study and the AAST2 study, although the mortality figures demonstrate support for endovascular repair, the results showed some areas of significant concern. In the AAST study, these concerns include device-related complications: $20 \%$ stent-graftrelated complications and $14.4 \%$ endoleak rates in the endovascular group. In addition, 6 patients with endoleaks eventually required conversion to open repair. The devicerelated complications are mainly due to the relatively small aortic diameter in these young patients and the acute angulation of the aortic arch, as opposed to elderly patients who typically have a greater aortic arch curvature due in part to the aging process, resulting in aortic elongation that is well suited to allow a more efficient stent-graft conformation.

We recently assessed causative factors of stent-graft collapse after thoracic endovascular aortic repair. ${ }^{13}$ This stentgraft-related complication seems related to poor apposition of the stent-grafts in the highly angulated aortic arch. Excessive stent-graft oversizing could be an additional causative factor. Several therapeutic options can be proposed. Most reported cases have been treated by the implantation of a giant Palmaz stent (Cordis, Miami Lakes, Fla) or by the implantation of another endovascular graft within the collapsed stent-graft. Open stent-graft explant and repair through a left thoracotomy as performed on our patient is another option. In a previous experimental study, ${ }^{14}$ we assessed fixation of 4 commercially available thoracic stentgrafts according to aortic arch angulation and to oversizing. The major features in stent-graft design providing a secure proximal anchorage seem to be the radial force and the presence of a proximal open stent segment. As a consequence, in cases of severe aortic arch angulation, a stent-graft with a more efficient proximal anchorage system and a more important radial force seems to be advocated to avoid poor apposition of the proximal edge of the stent-graft. Early-generation thoracic stent-grafts were designed as straight tubes for larger diameter vessels, as seen with degenerative aortic disease. Modern thoracic aortic stentgrafts are available ranging in diameter from 21 to $46 \mathrm{~mm}$. Current stent-graft design includes smaller diameter grafts ( $21 \mathrm{~mm}$ for aortas of $16 \mathrm{~mm}$ ), with $18 \mathrm{~F}$ to $20 \mathrm{~F}$ delivery systems for such grafts, allowing vascular accessibility through 6-mm iliofemoral vessels. However, in young patients, femoral access cannot always be achieved because vessels can be stretched. Aortic diameters of young patients would fall in the smaller range, with 21- to 22 -mm diameter grafts commonly used. Other off-the-shelf strategies are occasionally used with smaller abdominal aortic cuffs for smaller aortic sizes.

The design of stent-grafts has evolved, and new generations of thoracic devices have been introduced with improvements in conformability and accuracy of the deployment systems to accommodate the tight aortic arches of the typical patient with traumatic transection of the aorta. In our series, most of specific stent-graft complications occurred with the previous generation thoracic stent-grafts. The growing experience of most of team and the significant delivery system refinement (tip capture mechanism allowing an enhanced control of the thoracic stent-graft during deployment) will decrease the risk of partial or complete coverage of the supra-aortic trunks. In case of coverage of the supra-aortic trunks, as a first attempt, traction of the stent-graft by a low-pressure balloon should always be tried. In case of failure, transposition or creation of a bypass 
is a surgical alternative but could be too long and potentially cause ischemic cerebral injuries. Retrograde catheterization and "interposition" of a bare metal stent between the thoracic stent-graft and the aortic wall allow preservation of arch branch patency during thoracic endovascular aortic repair and stenting by focally displacing the stent-graft device. This technique, already described as the "chimney graft technique" by Ohrlander and colleagues, ${ }^{15}$ can lead to a rapid restoration of flow in case of inadvertent coverage of the supra-aortic trunks.

The main limitation of this study was the small sample size and its retrospective design. Even though long-term durability of endovascular repair of traumatic aortic transection remains unknown, endovascular repair provides better short- to midterm results. If needed, open repair can be performed until the patients fully recover from other injuries and can undergo an elective definitive open repair with proven long-term durability.

\section{CONCLUSIONS}

Compared with open repair, endovascular repair of traumatic thoracic aortic rupture is associated with a lower rate of death but failed to reach statistical significance most likely because of underpowering. These results prompt us to consider endovascular repair as the first-line therapy for acute traumatic rupture of the thoracic aorta. In some rare but challenging anatomic situations, such as acute aortic arch angulation, open surgical treatment should be discussed to avoid major perioperative or postoperative complications related to endovascular repair. Concerns over stent-graft durability are valid, but thus far results have been good. Furthermore, modern stent-grafts continue to evolve to accommodate the thoracic aorta, so long-term results should continue to improve. However, further studies and long-term survival studies are mandatory to determine the efficacy and durability of this technique.

\section{References}

1. Jahromi AS, Kazemi K, Safar HA, Doobay B, Cina CS. Traumatic rupture of the thoracic aorta: cohort study and systematic review. J Vasc Surg. 2001;34:1029-34.

2. Von Oppell UO, Dunne TT, De Groot MK, Zilla P. Traumatic aortic rupture: twenty-year meta-analysis of mortality and risk of paraplegia. Ann Thorac Surg. 1994;58:585-93

3. Dake MD, Miller DC, Semba CP, Mitchell RS, Walker PJ, Liddell RP. Transluminal placement of endovascular stent-grafts for the treatment of descending thoracic aortic aneurysms. N Engl J Med. 1994;331:1729-34.

4. Baker SP, O'Neill B, Haddon W, Long WB. The Injury Severity Score: a method for describing patients with multiple injuries and evaluating emergency care. J Trauma. 1974; 14:187-96.

5. Cook J, Salerno C, Krishnadasan B, Nichols S, Meissner M, Karmy-Jones R. The effect of changing presentation and management on the outcome of blunt rupture of the thoracic aorta. J Thorac Cardiovasc Surg. 2006;131:594-600.

6. Jahromi AS, Kazemi K, Safar HA, Doobay B, Cina CS. Traumatic rupture of the thoracic aorta: Cohort study and systematic review. J Vasc Surg. 2001;34:1029-34.

7. Fabian TC, Richardson JD, Croce MA, Smith JS Jr, Rodman G Jr, Kearney PA, et al. Prospective study of blunt aortic injury: Multicenter Trial of the American Association for the Surgery of Trauma. J Trauma. 1997;42:374-80.

8. Fabian TC, Richardson JD, Croce MA, Smith JS Jr, Rodman G Jr, Kearney PA, et al. Operative repair or endovascular stent-graft in blunt traumatic thoracic aortic injuries: results of an American Association for the Surgery of Trauma Multicenter Study. J Trauma. 2008;64:561-71

9. Xenos ES, Abedi NN, Davenport DL, Minion DJ, Hamdallah O, Sorial EE, et al. Meta-analysis of endovascular vs open repair for traumatic descending thoracic aortic rupture. J Vasc Surg. 2008;48:1343-51.

10. Peterson BG, Eskandari MK, Gleason TG, Morasch MD. Utility of left subclavian artery revascularization in association with endoluminal repair of acute and chronic thoracic aortic pathology. J Vasc Surg. 2006;43:433-9.

11. Buth J, Harris PL, Hobo R, van Eps R, Cuypers P, Duijm L, Tielbeek X. Neurologic complications associated with endovascular repair of thoracic aortic pathology: incidence and risk factors. A study from the European Collaborators on Stent/Graft Techniques for Aortic Aneurysm Repair (EUROSTAR) registry. J Vasc Surg. 2007;46:1103-10.

12. Botta L, Russo V, Savini C, Buttazzi K, Pacini D, Lovato L, et al. Endovascular treatment for acute traumatic transection of the descending aorta: focus on operative timing and left subclavian artery management. J Thorac Cardiovasc Surg. 2008;136:1558-63

13. Canaud L, Alric P, Desgranges P, Marzelle J, Marty-Ané C, Becquemin JP. Factors favoring stent-graft collapse after thoracic endovascular aortic repair. J Thorac Cardiovasc Surg. 2010;139:1153-7.

14. Canaud L, Alric P, Laurent M, Baum TP, Branchereau P, Marty-Ané CH, Berthet JP. Proximal fixation of thoracic stent-grafts as a function of oversizing and increasing aortic arch angulation in human cadaveric aortas. $J$ Endovasc Ther. 2008;15:326-34.

15. Ohrlander T, Sonesson B, Ivancev K, Resch T, Dias N, Malina M. The chimney graft: a technique for preserving or rescuing aortic branch vessels in stent-graft sealing zones. J Endovasc Ther. 2008;15:427-32. 